\title{
A Routing Protocol and Energy Efficient Techniques in Bluetooth Scatternets
}

\author{
Balakrishna J. Prabhu and A. Chockalingam \\ Department of Electrical Communication Engineering \\ Indian Institute of Science, Bangalore 560012. INDIA
}

\begin{abstract}
In this paper, we propose a protocol for routing in Bluetooth scatternets. The protocol uses the available battery power in the Bluetooth (BT) devices as a cost metric in choosing the routes. We evaluate the throughput performance as a function of packet arrival rate and number of piconets. A throughput of about $120 \mathrm{Kbps} /$ piconet is shown to be achieved in a 5piconet scatternet. We propose two techniques, namely a) battery power level based master-slave switch and b) distance based power control, to increase the network lifetime in scatternets. The master-slave switch technique is motivated by the fact that a piconet master has to handle the packet transmissions to/from all its slaves, and hence may drain its battery soon. We propose a role switching idea where each BT device in a piconet may have to play the master role depending on its available battery power. In the second technique, we propose that the BT devices choose their transmit powers based on their distances from their respective masters. Our performance results show that a considerable gain in network lifetime can be achieved using these two power saving techniques.
\end{abstract}

Keywords - Bluetooth scatternet, network lifetime, masterslave switch, power control.

\section{INTRODUCTION}

Bluetooth is a short-range $(\leq 10 \mathrm{~m})$, low-power ( 1 to 100 $\mathrm{mW}$ ) wireless technology to provide communications between various devices such as PDAs, cellphones, laptops and desktops [1],[2]. Bluetooth operates in the unlicensed $2.4 \mathrm{GHz}$ ISM band. It uses frequency hopped spread spectrum technique to alleviate the effects of interference. The system uses 79 carrier frequencies over $1 \mathrm{MHz}$ bandwidth. The nominal bit rate of transmission is 1 Mbps. Bluetooth allows a collection of devices to form small, overlapping networks (known as piconets) in an ad-hoc fashion. Each piconet can have upto 8 active Bluetooth (BT) devices. The device that establishes and coordinates a piconet is called the master. All other participants are called slaves. Time division duplexing (TDD) is used for the communication between the master and the slaves. The master transmits in the even numbered slots and the slaves transmit in the odd numbered slots.

A collection of piconets with overlapping coverage areas is called a scatternet. A slave can participate in more than one piconet (but at any given time, a device can be active in only one piconet). We call such devices as bridge points. The pres-

\footnotetext{
This research was supported in part by the Department of Science and Technology, Govt. of India, New Delhi, under scheme III.5(32)/99-ET.
}

ence of bridge points in scatternets makes inter-piconet communications possible. An example of a scatternet with four piconets is shown in Fig. 1. Devices $M_{1}, M_{2}, M_{3}, M_{4}$ are masters, $B_{12}, B_{13}, B_{14}, B_{23}, B_{34}$ are bridge points, and the rest are slaves. Topology formation and routing in scatternets are studied in [3]-[5].

In this paper, we propose a protocol for routing in Bluetooth scatternets. The protocol uses the available battery power in the Bluetooth devices as a cost metric for selecting the routes. We evaluate the throughput performance in scatternets as a function of packet arrival rate and number of piconets. A throughput of about $120 \mathrm{Kbps} /$ piconet is shown to be achieved in a 5-piconet scatternet.

A new contribution in this paper is the proposal of two energy saving techniques to increase network lifetime in scatternets. We consider all the devices in the scatternet, including the masters and the bridge points, to operate on finite-energy batteries. The proposed techniques exploit $a$ ) the master-slave switch option and $b$ ) power control capability (i.e., ability to vary the transmit power in steps), that are provided for in the standards (ref. [2], pp. 123, 20). The master-slave switch technique is motivated by the fact that a piconet master has to handle the packet transmissions to/from all its slaves, and hence may drain its battery soon. We propose a role switching idea, where each BT device in a piconet may have to play the master role depending on its available battery power. In the second technique, we propose that the BT devices choose their transmit powers based on their distances from their respective masters. We evaluate the network lifetime achieved using these two techniques through simulations. Our performance results show that a considerable gain in network life can be achieved using these two power saving techniques.

The rest of the paper is organized as follows. In Section II, we describe the routing protocol. The master-slave switch and power control techniques to increase network lifetime are described in Section III. Section IV provides the the simulation setup, results and discussions. Conclusions are given in Section V.

\section{Routing PRotocol For Scatternet}

A Bluetooth device has a unique 48-bit address known as the Bluetooth device address (BD_ADDR). At any given time, a slave in a piconet can be in any one of the following four 


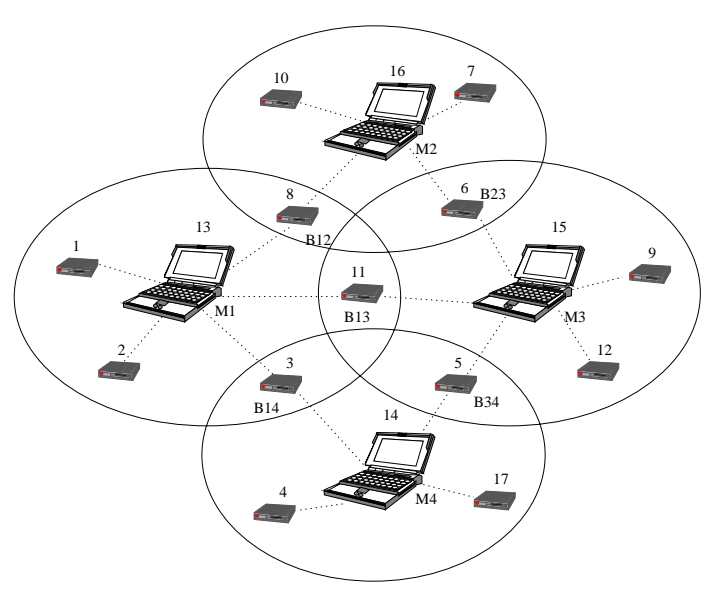

Fig. 1. An example of a Bluetooth scatternet with four piconets.

modes: active mode, sniff mode, hold mode, and park mode. In the active mode, a slave remains synchronized to its master and normal data transmission occurs between master and slave. The piconet master assigns a 3-bit Active Member address (AM_ADDR) to each of its active slaves. The sniff mode is a low power mode in which a the duty cycle of the slave's listen activity is reduced. That is, the slave listens for transmissions only in sniff slots which are spaced at a time interval of T_SNIFF (instead of listening every alternate slot as in the case of active mode). In the park mode, the slave does not participate in the piconet and releases its AM_ADDR. It, however, remains synchronized to the master by periodically waking up and listening to the master transmissions.

An active slave can enter the hold mode for various reasons, including inquiring, paging, attending other piconets, etc. We allow the bridge points to operate in the hold mode to participate in different piconets to enable inter-piconet communication. Prior to entering the hold mode, the master and the bridge point agree upon the time duration the bridge point will remain in the hold mode. We refer to this time duration as HOLD_TIME. After the HOLD_TIME time interval, the bridge point once again comes back to active mode.

\section{A. Routing Protocol}

We assume that a scatternet has been formed using a topology formation protocol, such as the one mentioned in [3],[4], and during the scatternet formation each piconet is given a unique piconet ID (PID). When a device wants to discover a route to another device in the scatternet, it sends a ROUTE_REQ (route request) packet to its master. The master appends its PID and its available battery power level (cost field) to the request packet and forwards it to all the associated bridge points. Each bridge point will append its own BD_ADDR and add its available battery power level to the cost field and forward the packet to all other piconets that it

\begin{tabular}{|c|l|}
\hline BD_ADDR & Routing Vector \\
\hline 13 & $1(\mathrm{P})$ \\
\hline 3 & $1(\mathrm{P}), 3(\mathrm{~B})$ \\
\hline 14 & $1(\mathrm{P}), 3(\mathrm{~B}), 4(\mathrm{P})$ \\
\hline 5 & $1(\mathrm{P}), 3(\mathrm{~B}), 4(\mathrm{P}), 5(\mathrm{~B})$ \\
\hline 15 & $1(\mathrm{P}), 3(\mathrm{~B}), 4(\mathrm{P}), 5(\mathrm{~B}), 3(\mathrm{P})$ \\
\hline
\end{tabular}

TABLE I

AN EXAMPLE OF ROUTE DISCOVERY

is associating with. This process continues until the request packet reaches the destination device. The destination may get multiple copies of the ROUTE_REQ packet through different routing paths having different costs. The destination waits for a specified amount of time to get multiple copies of the ROUTE_REQ packet through different paths. It then selects the path which has the maximum cost field (i.e., maximum cumulative battery power in the path). The destination then sends a ROUTE_REP (route reply) packet to the source device. The ROUTE_REP packet contains the selected route vector. Subsequent data packets flow from the source to destination will follow this chosen route.

Figure 1 shows an example scatternet with four piconets. The nodes with labels $M_{1}, M_{2}, M_{3}$, and $M_{4}$ indicate the masters of the piconets which have been assigned PIDs 1, 2, 3, and 4, respectively. In Fig. 1, the number shown alongside each device is the device's BT device address. Consider a ROUTE_REQ packet generated by the source device with BD_ADDR=1 meant to find a route to the destination device with BD_ADDR=9 in the piconet with $\mathrm{PID}=4$. One of the paths taken by this packet is given in Table I. The entries in this Table are explained as follows. In each row of the Table, the routing vector field is shown as it will be when the packet leaves the device having BT device address, BD_ADDR. For example, the device with BD_ADDR=13 is the master of piconet with PID=1, and hence the master $M_{1}$ adds its PID of 1 to the routing vector. This is shown in the first row of the Table. Next, the bridge point $B_{14}$ with BD_ADDR=3 appends its BD_ADDR to the vector, as shown in the second row. This procedure continues until the ROUTE REQ packet reaches the destination. Note that the ' $\mathrm{P}$ ' or ' $\mathrm{B}$ ' inside the parenthesis () in the route vector indicates whether the address is a PID or BD_ADDR. Also, note that the first and the last entries in the routing vector are always PIDs. However, the intermediate entries could be the BD_ADDR of the bridge points in the route.

\section{ENERGy EFFICIENT TeChNiQues}

In this Section, we present two techniques which can save energy in BT devices and hence can improve network lifetime in scatternets. We define network lifetime as the time it takes 
till any BT device in the network exhausts all its battery power. We propose to use the master-slave switch option as well as the capability to control the transmit power in steps, which are provided for in the standard. We base the master-slave switch on the available battery power levels in the devices, and base the power control on the distance between the master and the slave.

\section{A. Battery Level Based Master-Slave Switch}

The master-slave switch technique is motivated by the fact that a piconet master has to handle the packet transmissions to/from all its slaves. If we consider all the devices in the scatternet, including the piconet masters, to operate on finiteenergy batteries, then the masters may drain their batteries sooner than the slaves. The slaves may have substantial residual battery energy after the master runs down its battery completely. In order to achieve a more uniform residual battery energy profile and to increase the network lifetime, we propose a role switching idea where each BT device in a piconet may have to play the master role depending on its available battery power. That is, a master in a piconet is dynamically chosen based on the available battery power.

The proposed available battery level based master-slave switch procedure is described as follows. The current master in a piconet periodically monitors its own as well its slaves' available battery power levels. If its own battery power is less than a fraction $X(0<X<1)$ of the maximum available battery power amongst its slaves, then it initiates a masterslave switch procedure with the slave having the maximum battery power, as described in [2] (ref. pp123). The slave then assumes the role of the master, and informs all the devices about the role switch. The new master then will start periodically checking for the master-slave switch criterion to be satisfied. When the criterion is satisfied another master-slave switch will occur. This process will continue. It is noted that a finite time gets elapsed in completing a master-slave switch. Frequent master-slave switches can thus degrade the system performance. In this study, we assume that all the nodes in a piconet are within listening distance of each other so as to avoid reconfiguration of the topology every time a switch takes place. Further, it is assumed that at the time of the switch, a bridge point is in active mode in the piconet where the switch occurs. This ensures that the bridge points are aware of the switch.

\section{B. Distance Based Power Control}

In the Bluetooth specifications, the option of controlling the transmit power of the Bluetooth devices is steps has been provided [2]. Power control can be used not only to reduce interference but also to extend the life of battery in a device. The standards define three power classes each with a different transmit power range. Transmit power step sizes in the range 2 to $8 \mathrm{~dB}$ have been specified. We propose to choose the transmit power of the master/slave based on the distance, $d$, between the master and the slave. It is assumed that the distance between a master and a slave is known both to the master and the slave. The distance loss is proportional to $d^{\eta}$, where, in line-of-sight indoor environments, $\eta$ is typically taken to be 2 [6]. Using this distance loss model we devise the power control strategy in such a way that the transmitter chooses its transmit power based on distance, according to Table II.

\begin{tabular}{|c|c|}
\hline Distance, $d(\mathrm{~m})$ & Tx. Power \\
\hline$d \leq 1$ & $0 \mathrm{dBm}$ \\
\hline $1<d \leq 2$ & $6 \mathrm{dBm}$ \\
\hline $2<d \leq 4$ & $12 \mathrm{dBm}$ \\
\hline $4<d \leq 8$ & $18 \mathrm{dBm}$ \\
\hline $8<d \leq 10$ & $20 \mathrm{dBm}$ \\
\hline \multicolumn{2}{|c|}{} \\
\hline
\end{tabular}

TRANSMIT POWER AS A FUNCTION OF DISTANCE

\section{Simulation Results and Discussion}

In this Section, we first present the throughput performance in Bluetooth scatternets as a function of number of piconets and packet arrival rate. The routes are assumed to be chosen based on the routing protocol presented in Section II. We then present the network lifetime performance of the energy efficient techniques presented in Section III.

The following simulation model is used for the throughput performance evaluation. It is assumed that a scatternet has been formed (using a topology formation protocol) and each piconet has been given a unique PID. During the network startup, all devices are assumed to have an initial energy of 50000 units. Each packet is transmitted with the maximum allowed transmit power (i.e., there is no power control based on distance). One unit of energy corresponds to the energy consumed due to a packet transmission in a slot. Also, the energy expended in packet reception in a slot is taken to be $10 \%$ of the energy expended in packet transmission in a slot. Each slot can carry 500 information bits (excluding overhead bits). Each DATA packet is assumed to occupy 5 slots. ROUTE_REQ and ROUTE_REP packets are assumed to occupy 1 slot each. The HOLD_TIME is expressed in number of slots.

Fig. 2 shows the system throughput as a function of packet arrival rate (packets/sec/node) and the HOLD_TIME, for a 5piconet scatternet with 23 BT devices. The plots are parameterized by the HOLD_TIME values of 20,40, 60, and 80 slots. The throughput is defined as the amount of data bits (excluding overhead bits) successfully delivered end-to-end, per unit time. The throughput plotted in Fig. 2 is the overall system throughput across all the piconets in the scatternet. 
The per-piconet throughput can be obtained by normalizing the system throughput by the number of piconets. It is observed that, as expected, the throughput increases as the packet arrival rate increases. When HOLD_TIME $=20$ slots, a system throughput of about $550 \mathrm{Kbps}$ is achieved implying a perpiconet throughput of about $110 \mathrm{Kbps} /$ piconet. It is also noted that, for a given packet arrival rate, throughput increases as the HOLD_TIME value is increased. This is because, if the HOLD_TIME is small then the chances of the bridge point exchanging intra-piconet packets will be less, and this can result in reduced throughput. As the HOLD_TIME is increased, more inter-piconet traffic can flow between the bridge point and the master leading to increased throughput. In the limits when HOLD_TIME $\rightarrow 0$ or HOLD_TIME $\rightarrow \infty$, the bridge point will tend to become a full time slave to a particular piconet and cease to carry inter-piconet traffic. The throughput, in such cases, would be mainly due to intra-piconet traffic, and the delay for inter-piconet traffic will become prohibitively large. Thus, an optimum choice of the value of HOLD_TIME needs to be made for efficient transfer of inter-piconet traffic. Considering that a bridge point can be one of the seven slaves in a piconet, and that 5 slots per data packet is considered (i.e., a full duplex transfer of 10 slots; 5 slots in the master-to-slave direction and 5 slots in the slave-to-master direction), we have used HOLD_TIME $=80$ slots as a reasonable choice in all our subsequent simulations.

Fig. 3 shows the per-piconet throughput (in Mbps/piconet) as a function of number of piconets and packet arrival rate. We see that a throughput of $450 \mathrm{Kbps}$ is achieved in a singlepiconet system at high arrival rates. This is about one half of the transmission rate of $1 \mathrm{Mbps}$, due to the TDD mode of operation between the master and the slaves and the overhead bits. As the number of piconets increase, the throughput per piconet comes down for a given packet arrival rate. This is because of the multiple hops that a packet may have to take for inter-piconet communications. For example, the per-piconet throughput achieved in a 5-piconet scatternet is about $130 \mathrm{Kbps}$ compared to $450 \mathrm{Kbps}$ throughput achieved in a single-piconet case.

Next, we illustrate the network lifetime and the number of packets delivered performance in Figs. 4 and 5, respectively, as a function of packet arrival rate with and without the distance based power control described in Section III.B. The number of piconets considered is 4 , and the total number of devices is 20. It is observed that the network lifetime achieved with power control is more compared to that without power control. This is because power control reduces the energy spent in the packet transmission. Also the network lifetime reduces as the arrival rate increases (with or without power control). This is because at high load, devices may always have packets to send thus depleting the batteries faster. However, the number of packets sent during the lifetime increases as the arrival rate increases, as seen from Fig. 5. Also, the number

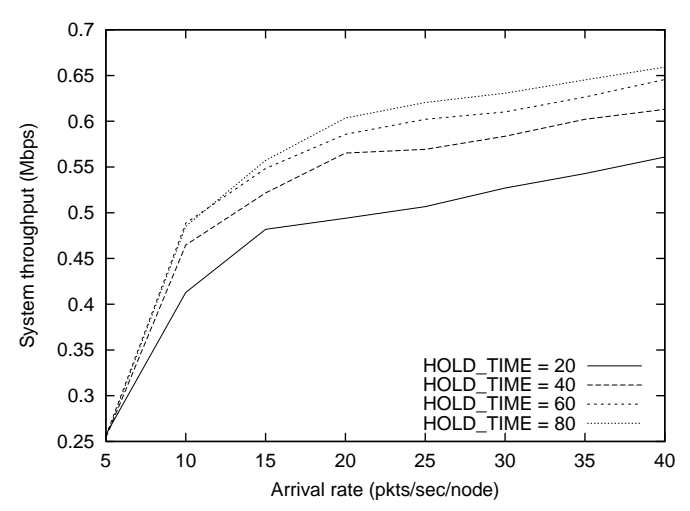

Fig. 2. System throughput versus packet arrival rate for different values of HOLD_TIME. Number of piconets $=5$. Number of nodes $=23$.

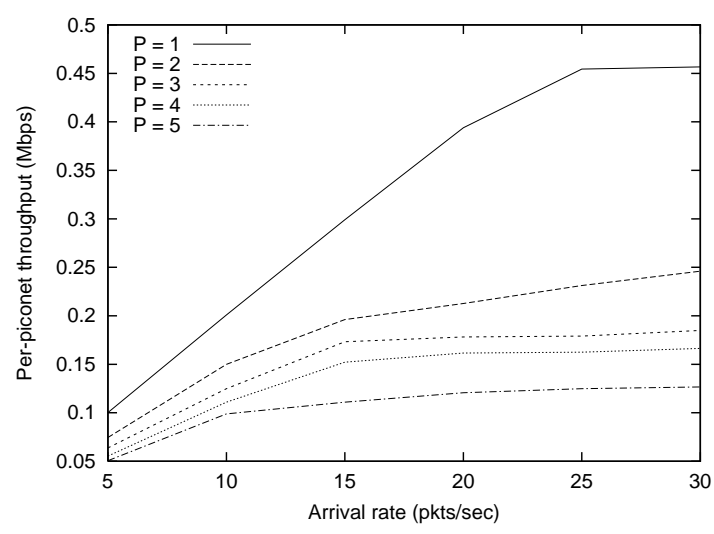

Fig. 3. Per-piconet throughput versus packet arrival rate for different values of number of piconets, P. HOLD_TIME $=80$ slots

of packets delivered with power control is more compared to that without power control. Note that the system throughput can be obtained from figs. 4 and 5 by computing the number of packets delivered per unit network lifetime times the number of data bits per packet, which turns out to be almost the same with and without power control. This means that power control achieves the same throughput as without power control but for an extended network lifetime.

Finally, the performance of the battery power level based master-slave switch is illustrated in Figs. 6 and 7. The network lifetime and number of packets delivered are plotted as a function of the master-slave switch factor, $X$. It has been assumed that a master-slave switch operation does not alter the existing topology, except for the master-slave role changes. In the simulations, the time taken for a master-slave switch to complete is taken to be 50 slots. Also, we have taken the energy expended during the master-slave switch operation by the new master, the old master, and the slaves, to be 15, 3, and 2 units of energy, respectively. The new master is assumed to consume more energy because it has to inform all slaves about 


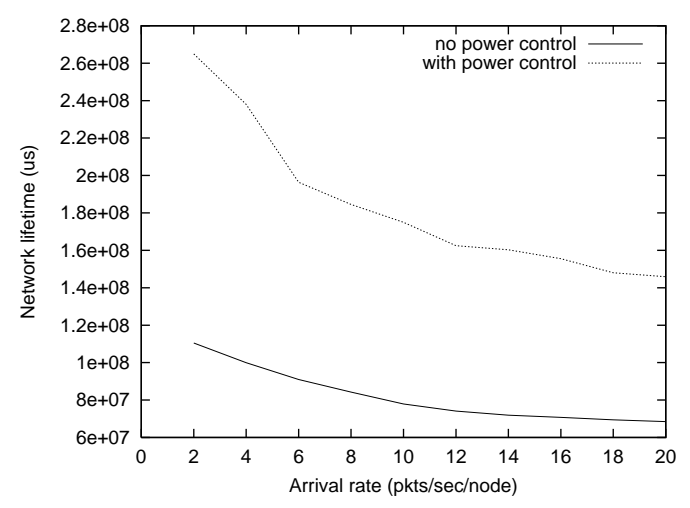

Fig. 4. Network lifetime versus packet arrival rate with and without power control. Number of piconets $=4$. Number of nodes $=20$. HOLD_TIME $=80$ slots.

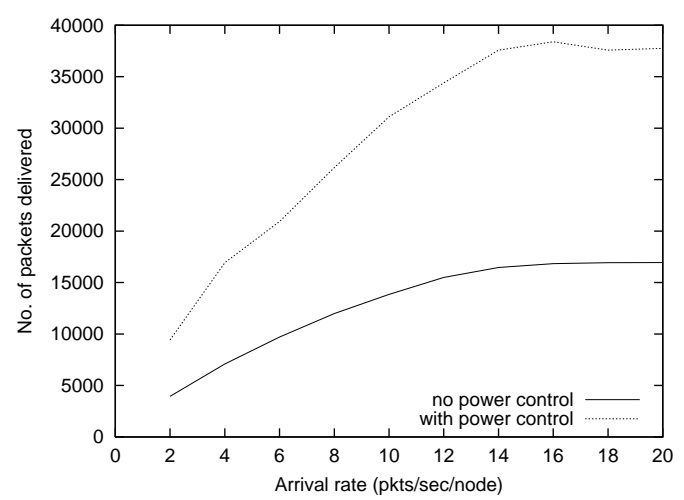

Fig. 5. Number of packet delivered versus packet arrival rate with and without power control. Number of piconets $=4$. Number of nodes $=20$. HOLD_TIME $=80$ slots.

the switch. From Figs. 6 and 7, we observe that the network lifetime increases significantly due to the master-slave switch. Note that $X=0$ corresponds to no master-slave switch. As $X$ is increased, the rate at which the switch occurs is increased resulting in increased lifetime. As the threshold, $X$, increases the number of switches increase which can lead to a degradation of the throughput performance. For the scenario we simulated, the bridge points exhausted their energy before many switches could take place at higher values of $X$, and hence there is only a small degradation in throughput performance.

\section{Conclusions}

In this paper, we proposed a protocol for routing in Bluetooth scatternets. The protocol used the available battery power in the Bluetooth devices as a cost metric in choosing the routes. We evaluated the throughput performance as a function of packet arrival rate and and number of piconets. We showed that a throughput of about $120 \mathrm{Kbps} /$ piconet is achieved in a 5-piconet scatternet. We also proposed two energy saving

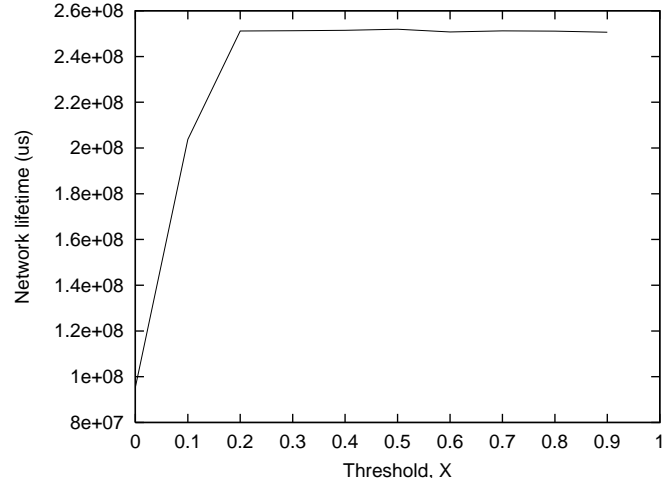

Fig. 6. Network lifetime versus master-slave switch parameter, $X$. Number of piconets $=4$. Number of nodes $=20$. Packet arrival rate $=5 \mathrm{pkts} / \mathrm{sec} /$ node . HOLD_TIME $=80$ slots.

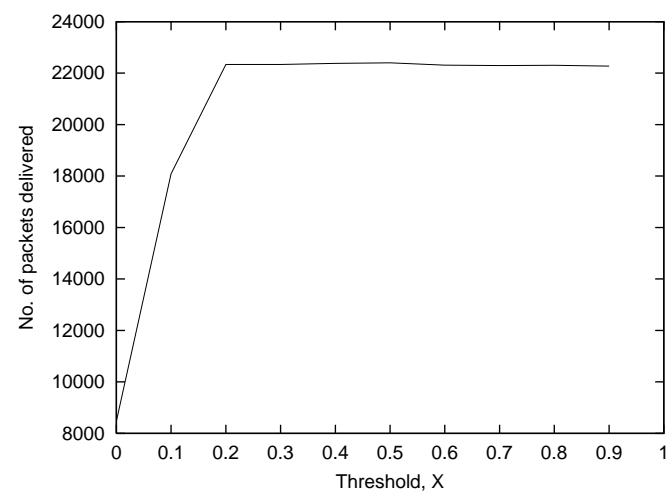

Fig. 7. Number of packet delivered versus master-slave switch parameter, $X$. Number of piconets $=4$. Number of nodes $=20$. Packet arrival rate $=5$ pkts/sec/node. HOLD_TIME $=80$ slots.

techniques; one using available battery power based masterslave switch and the other using distance based power control, to increase the network lifetime in scatternets. Through simulations, we showed that a considerable gain in network life can be achieved using these two power saving techniques.

\section{REFERENCES}

[1] J. C. Haartsen, "The Bluetooth Radio System," IEEE Personal Commun. Mag., pp. 28-36, vol. 7, February 2000.

[2] Bluetooth SIG, Bluetooth Baseband Specifications Version 1.OB. http://www.bluetooth.com

[3] T. Salonidis, P. Bhagwat, L. Tassiulas, and R. LaMaire, "Distributed topology construction of Bluetooth personal area networks," Proc. IEEE INFOCOM'2001, 2001.

[4] G. V. Zaruba, S. Basagni, and I. Chlamtac, "Bluetrees - Scatternet formation to enable Bluetooth-based ad-hoc networks," Proc. IEEE ICC'2001, Helsinki, June 2001.

[5] P. Bhagwat and A. Segall, "A routing vector method (RVM) for routing in Bluetooth scatternets," The Sixth IEEE Intl. Workshop on Mobile Multimedia Communications (MOMUC'99), November 1999.

[6] A. Karnik and A. Kumar, "Performance analysis of the Bluetooth physical layer," IEEE Intl. Conf. on Wireless Personal Commun. (ICPWC'2000), Hyderabad, December 2000. 